\title{
El sistema de apoyo internacional para la gestión forense de cadáveres en situaciones de desastre. La experiencia de Haití, 2010
}

\author{
The system of international support for the forensic management \\ of corpses in mass disasters situations. The Haiti experience, 2010
}

\section{Resumen}

Se presenta la experiencia de un grupo de expertos del Instituto de Medicina Legal y Ciencias Forenses de Panamá que acudió a la capital de Haití para ofrecer su apoyo en el manejo de cadáveres del gran terremoto ocurrido el 12 de enero de 2010. Al sistema forense del país afectado le corresponde el primer nivel de coordinación, del cual dependerá la aplicación de los principios forenses desarrollados a nivel internacional para este tipo de situaciones. Se plantea la necesidad de fortalecer las estructuras forenses nacionales y la implementación de las resoluciones aprobadas en el seno de la Red Iberoamericana de Institutos Nacionales de Medicina Legal y Ciencias Forenses.

Palabras clave: Medicina Legal. Desastres masivos. Terremoto en Haití. Manejo de cadáveres.

\section{Abstract}

In this paper, we present the experience of a group of experts from the Institute of Legal Medicine and Forensic Sciences of Panama, who went to Haiti to offer theirs support in the management of corpses of the great earthquake occurred in January 12th 2010. The forensic system of the country affected is the first level of coordination being responsible for the application of forensic principles developed internationally for such situations. The need to strengthen national forensic structures and the implementation of the resolutions adopted at the Latin American Network of National Institutes of Legal Medicine and Forensic Sciences are discussed.

Key words: Legal Medicine. Mass Disasters. Earthquake in Haiti. Management of dead bodies.

\section{Introducción}

La gestión de cadáveres en situaciones de desastre ha sido motivo de diferentes análisis y protocolos oficiales de circulación internacional. En los manuales elaborados para este tipo de situaciones, se afirma que, la gestión adecuada de los cadáveres es uno de los aspectos más difíciles de resolver en una situación de catástrofe; especialmente si se trata de calamidades naturales con incontable número de víctimas fatales cuando se hacen evidentes las limitaciones de la capacidad de respuesta1,2,3

Los desastres pueden ser de tal magnitud que los recursos locales para atender los cadáveres son insuficientes. La ausencia de planificación especializada en estas situaciones magnifica la gravedad de los múltiples problemas que acompañan al siniestro y la manera en la cual se traten los cadáveres tendrá un efecto profundo en la sociedad, especialmente en la memoria de los sobrevivientes.

La administración de los cuerpos debe hacerse de manera organizada evitando prácticas basadas en conceptos erróneos o prejuicios, como la inexacta opinión de que se pueden originar epidemias a consecuencia de la descomposición cadavérica, juicio equivocado que conduce a conductas que pueden ser consideradas extremas, como la quema de los cadáveres y los entierros masivos.

Por otra parte, la correcta identificación de los difuntos tiene serias consecuencias familiares, psicológicas,

\section{JV. Pachar ${ }^{1}$ \\ K. Bryan²}

${ }^{1}$ MPhil Forensic Pathology, Instituto de Medicina Legal y Ciencias Forenses de Panamá.

²Odontóloga Forense, Instituto de Medicina Legal y Ciencias Forenses de Panamá.

Correspondencia: Dr. José V. Pachar Lucio E-mail:

jvpachar@gmail.com

Fecha de recepción: 3-MAR-2010

Fecha de aceptación: 4-MAR-2010 
legales y sociales que, de no ser adecuadamente resueltas, acarrean graves repercusiones a futuro. Es por eso que se recomienda que la tarea de identificación de los cuerpos, proceso basado en criterios científicos, deba corresponderle a expertos nacionales, apoyados en la medida de lo necesario por la magnitud del desastre por expertos de otros países ${ }^{1,2}$.

A fin de obtener un resultado satisfactorio en la identificación, es necesario que, en la anarquía posterior al evento, se establezca un orden siguiendo planes de trabajo previamente elaborados, aplicables a diferentes niveles, local, regional, nacional y regional.

La principal estructura u organización conductora del país afectado, deberá activarse lo más pronto posible y comenzar a realizar las tareas de manejo de la información y coordinación de actividades que implica la movilización de los equipos de trabajo, las tareas administrativas y de logística asociados a la implementación de un plan estratégico para el manejo de los cadáveres. Posteriormente, la coordinación principal deberá organizar la divulgación de la información a los familiares y a la sociedad con datos periódicos y precisos del proceso de identificación de los cuerpos.

Un manejo adecuado de los cadáveres en situaciones de desastre con numerosas víctimas fatales requiere de coordinación local, regional, nacional e internacional. A nivel internacional, es importante mencionar la participación de las Naciones Unidas, de la Organización Mundial de la Salud, de la Organización Panamericana de la Salud, del Comité Internacional de la Cruz Roja, de la Federación Internacional de las Sociedades de la Cruz Roja, de la Media Luna Roja y de Interpol.
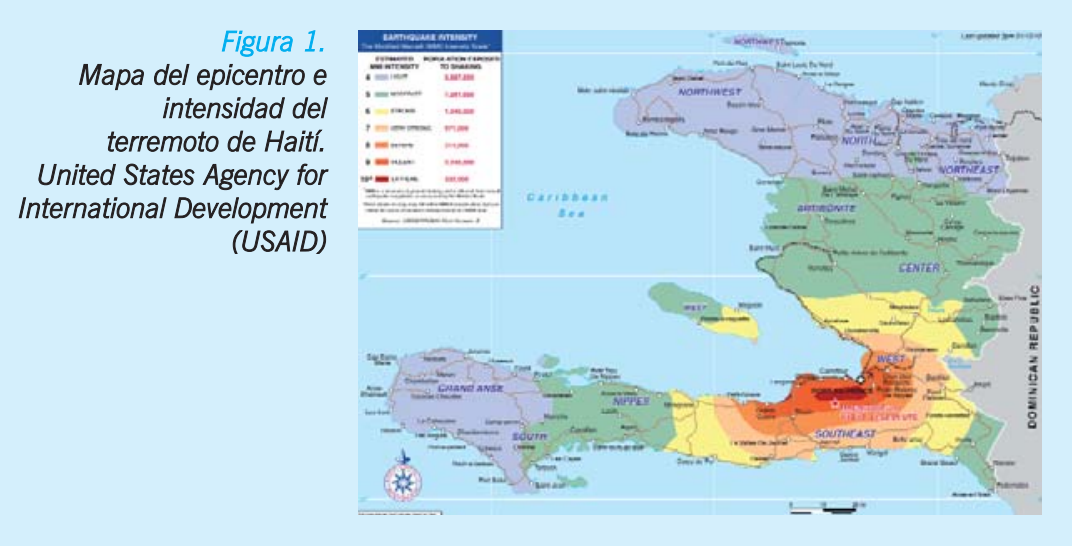

\section{La experiencia haitiana}

El 12 de enero de 2010, a las 16:53:09 hora local, con epicentro a $15 \mathrm{~km}$ de Puerto Príncipe, ocurrió un seísmo de 7,3 grados de magnitud, generado a una profundidad de 10 kilómetros. El sismo fue perceptible en países cercanos como Cuba, Jamaica y República Dominicana, donde provocó temor y evacuaciones preventivas ${ }^{4}$.

Los efectos causados sobre el país más pobre de América Latina han sido devastadores. Un informe del Banco Interamericano de Desarrollo (BID), reveló que el seísmo superó en destrucción al tsunami del año 2.004 en el Océano Índico calculándose que el número de muertos podría sobrepasar los 217.000. También habría producido más de 250.000 heridos y dejado sin hogar a un millón de personas. Se considera una de las catástrofes humanitarias más graves de la historia ${ }^{5}$. En la Figura 1 se ilustra la intensidad del terremoto.

El terremoto afectó principalmente la capital, Puerto Príncipe, causando cuantiosos daños materiales y miles de heridos y víctimas mortales. Inmediatamente se movilizaron equipos internacionales de rescate para buscar sobrevivientes entre los escombros.

En la etapa inmediatamente posterior, la capital quedó prácticamente incomunicada, sin servicios públicos básicos; había una situación de anarquía social y era casi imposible circular por las calles destruidas e invadidas por los escombros. Los daños materiales fueron masivos en los edificios de la ciudad, debido al tipo y estructura de las construcciones; incluso la catedral se derrumbó, y el palacio presidencial sufrió daños severos.

Un grupo de forense panameños, conformado por la Odontóloga Forense, Dra. Karina Bryan, el Médico Forense, Juan Carlos Rodríguez y el Patólogo Forense, José Vicente Pachar Lucio, se trasladó hasta la zona del desastre en Haití, dando cumplimiento a los acuerdos logrados en las reuniones de la Red Iberoamericana de Institutos Nacionales de Medicina Legal y Ciencias Forenses; encuentros celebrados con el propósito de crear un sistema de apoyo a nivel internacional en situaciones de desastre. Las propuestas de la Red apuntan al noble objetivo del apoyo mutuo internacional que tanto requiere la diversidad de circunstancias, propias del desarrollo social, político y científico de cada país en nuestro campo forense.

Los objetivos de nuestra participación eran: colaborar en la identificación y traslado de posibles víctimas panameñas e incorporarnos a los equipos 
internacionales para apoyar la gestión de cadáveres en situaciones de desastre.

Al llegar a Puerto Príncipe, a cuatro días de ocurrido el siniestro, y debido a la situación de caos total, fue imposible hacer contacto con ninguna coordinación local o internacional, confrontando serios problemas por las réplicas del temblor y la anarquía reinante pero sobre todo por la falta de seguridad y la ausencia de autoridades coordinadoras; ellas mismas también profundamente afectadas en gran medida por la muerte de sus funcionarios, la destrucción de sus edificios sede, y muchas veces por la pérdida de sus familiares, amigos y de sus pertenencias.

Uno de los obstáculos más importantes fue justamente el colapso o dificultades de acceso a los sistemas de comunicación, telefónicos y vía Internet.

Las autoridades consulares de Panamá en Haití nos informaron que no hubo víctimas fatales de nuestro país y debido a que no se habían dado las condiciones mínimas para cumplir con el segundo objetivo retornamos a Panamá.

\section{Discusión}

Un desastre, entendido como una situación catastrófica en la que han ocurrido cuantiosas pérdidas humanas y materiales ocasionadas por eventos relacionados a procesos naturales o por actividades humanas, es un proceso, dentro del cual es posible diferenciar y relacionar tres fases ${ }^{6}$ :

a. Antes: Situación inicial de riesgo.

b. Durante: Concreción del riesgo en el propio desastre propiamente tal, predominan las acciones de respuesta inmediata, como las tareas de rescate y atención a los sobrevivientes, el trabajo forense y el comienzo de las acciones de rehabilitación.

c. Después: Fase en que las autoridades y la población aplican la capacidad de acción recuperada para hacer frente a las "secuelas" del desastre. Predominan objetivos de reconstrucción y se plantea la posibilidad de darles un enfoque de desarrollo sostenible.

Múltiples son los abordajes e implicaciones que se dan en una situación de desastre a consecuencia de un terremoto capaz de causar devastación de la magnitud ocurrida en Haití.

La recuperación, los procedimientos de identificación y las recomendaciones de disposición de los cuerpos les corresponden a los expertos en Medicina y Ciencias Forenses siendo determinante, en el resultado final, la pronta respuesta que se dé en el primer nivel de coordinación.

La recuperación de los cadáveres es el primer paso $y$, generalmente, dadas las condiciones imperantes, es anárquica y confusa. Muchos voluntarios y organizaciones se encuentran involucrados en la pronta recuperación de los fallecidos. Con frecuencia, en los primeros días consecutivos al desastre, es difícil comunicarse y coordinarse entre ellos, dificultad en este caso extensiva a los expertos internacionales quienes acuden voluntariamente a respaldar las labores forenses.

Una vez recuperados los cadáveres, el cumplimiento de las recomendaciones consignadas en los manuales preparados sobre el tema es esencial para una identificación eficaz y eficiente. Esta fase puede durar días o semanas, dependiendo de la dimensión de la tragedia. Así mismo es muy importante tomar en consideración los problemas de logística, administrativos, sanitarios y de seguridad para el personal forense. Los cadáveres deben ser depositados considerando las distintas opciones de almacenamiento, incluyendo la sepultura temporal.

La identificación de los cuerpos es un complejo proceso de confrontación de datos de la persona fallecida con la información disponible sobre personas desaparecidas; incluyendo los elementos más simples y menos confiables como el reconocimiento visual corporal, del vestuario, de las prendas y de algunas características particulares como cicatrices, tatuajes y prótesis. Dada la magnitud del desastre, generalmente la mayor parte de los casos se resuelven en base a la presencia de esos elementos; sin embargo, la identificación científicamente positiva de casos más complejos debe realizarse mediante estudios antropológicos, la comparación de huellas dactilares, el análisis odontológico forense y la prueba de $\mathrm{ADN}^{2}$.

El laborioso proceso de identificación debe hacerse siguiendo una serie de pasos que incluye la asignación de un número único de referencia a cada cadáver, el etiquetado y la fotografía con su correspondiente registro ${ }^{7}$.

En el caso de Haití, la información disponible sobre la infraestructura y recursos humanos en materia de Medicina Forense es bastante limitada y solamente disponemos de documentos de las Naciones Unidas, en su Índice Universal de Los Derechos Humanos, que recomiendan la creación de un Instituto de Medicina Forense, con la separación de las dos morgues principales, la del Hospital estatal de la del Instituto 
Medicolegal. También se recomienda la extensión de los servicios a las jurisdicciones provinciales por medio de la creación de al menos dos agencias del Instituto en las ciudades de Le Cap y Les Cayes ${ }^{8}$.

Las únicas referencias encontradas hacen énfasis en los crímenes y las sistemáticas violaciones a los derechos humanos ocurridas en los últimos años relacionadas con las condiciones sociopolíticas del país; sin embargo no encontramos un registro proveniente de una institución médico legal organizada ni de la cuantificación de los recursos disponibles ${ }^{8,9}$.

Una vez ocurrido el cataclismo, la poca capacidad de respuesta forense local organizada fue completamente anulada y la anárquica situación del país, sumada a la falta de comunicaciones y seguridad, impidió una adecuada coordinación con los equipos de otros países.

Esta experiencia contrasta, por ejemplo, con los resultados logrados por los equipos forenses internacionales en el tsunami de diciembre de 2.004 cuando de un grupo de 4.280 cadáveres más del $92 \%$ de las víctimas extranjeras y $80 \%$ de nacionales tailandeses fueron positivamente identificados mediante el examen odontológico forense ${ }^{10}$.

\section{Conclusiones y recomendaciones}

- La quema de cuerpos en las vías públicas y el entierro masivo de miles de cuerpos no identificados, la mayoría probablemente de ciudadanos haitianos, no cumplió con las normas internacionalmente impulsadas para este propósito; disposiciones tendientes a garantizar un trabajo científico, pero sobre todo para mitigar el sufrimiento psíquico de los sobrevivientes; al facilitar la recuperación, por las familias, de sus cadáveres debidamente identificados y, por ende, acceder al consuelo que aportan los rituales de duelo propios, que en este hecho hubieran sido los de una cultura tan fecunda y compleja como la haitiana.

- La carencia de servicios forenses locales organizados, en el nivel requerido para atender un desastre masivo, propició la desorganización y la falta de coordinación necesarias para acoger el apoyo que hubieran podido ofrecer los equipos especializados que acudieron de otros países.

- Quizás en situaciones similares un rol apropiado para la Red Iberoamericana de Institutos Nacionales de Medicina Legal y Ciencias Forenses sería el de ofrecer apoyo en la etapa anterior a un posible desastre, apuntalando la preparación que fortalezca el liderazgo local en los primeros días del desastre y que apunte a subsanar, mediante un respaldo al desarrollo forense sostenido, las insuficiencias administrativas, de logística y operativas que en esta ocasión hicieron poco productiva la disponibilidad de los expertos que se desplazaron hasta allí.

- La experiencia debe ser compartida en foros de discusión sobre desastres como es el caso de la Red Iberoamericana de Institutos Nacionales de Medicina Legal y Ciencias Forenses.

\section{Agradecimientos}

Agradecemos la revisión del artículo a la Dra. Mary Luz Morales, Patóloga Forense de Colombia, con quien hemos compartido algunos avatares de la práctica forense. Ella tuvo la oportunidad de acudir a Haití en el contexto del apoyo ofrecido a este país por el Comité Internacional de la Cruz Roja (CICR) con ocasión del terremoto.

\section{Bibliografía}

1. La gestión de cadáveres en situaciones de desastre: Guía práctica para equipos de respuesta. Morgan Oliver, ed.; Tidball-Binz, Morris, ed.; Alphen, Dana van, ed. Washington, DC (USA); 2006. http://www.helid.desastres. net.

2. Manejo de cadáveres en situaciones de desastre Manuales y Guías sobre Desastres, N 5. Organiza- ción Panamericana de la Salud. Washington DC, agosto 2004. http://www.paho.org/spanish/ dd/ped/ManejoCadaveres/ntro.pdf.

3. Guía de identificación de victimas de catástrofes. Organización Internacional de Policía Criminal. Interpol 1997. http://www.interpol.int/Public/DisasterVictim/ guide/GuideEs.pdf.

4. USGS (12-01-2010). «Reporte preliminar: Magnitud 7,0 REGIÓN DE HAIT|». Consultado el 12-01-2010. 
5. Informe del Banco Interamericano de Desarrollo (BID) 123 CL. Entel internet. Entel SA. Febrero 2010.

6. Campos SA. Educación y Prevención de Desastres, 2000. En Red de Estudios Sociales en Prevención de Desastres en América Latina. http://www.desenredando.org/public/libros/2000/eypd/

7. Cordner S, Sribanditmongkol P. Identificación de los cadáveres, Capítulo 6. La gestión de cadáveres en situaciones de desastre: Guía práctica para equipos de respuesta. Tindball-Binz, Morris; Van Alphen, Dana Eds. Washington DC, 2006. http://www.helid.desastres.net
8. Índice Universal de Los Derechos Humanos. Sobre los documentos de las Naciones Unidas. http://www.universalhumanrightsindex.org/es/index. html.

9. Kolbe AR, Hutson RA. Human rights abuse and other criminal violations in Port-au-Prince, Haiti: a random survey of households. Lancet 2006;368:864-73.

10. Schuller-Götzburg P, Suchanek J. Forensic odontologists successfully identify tsunami victims in Phuket, Thailand. Forensic Sci Int 2007; 171(2-3):204-7. 